\title{
Octreotide-Induced Hypoglycemia in a Cirrhotic Patient
}

\author{
Debbie Chen, MD
}

\begin{abstract}
INTRODUCTION
Hepatorenal syndrome (HRS) is characterized by functional renal impairment in patients with end-stage liver cirrhosis and no parenchymal kidney disease. It is due to splanchnic vasodilation, which results in renal vasoconstriction with consequent reduction in renal plasma flow and glomerular filtration rate. ${ }^{1}$ In the United States, treatment is with a combination of octreotide, midodrine, and human albumin to increase the effective blood volume and subsequently improve renal function.? While octreotide, a long-acting somatostatin analog, is used to induce splanchnic vasoconstriction in this setting, it also affects the secretion of insulin and glucagon.
\end{abstract}

\section{CASE PRESENTATION}

A 44-year-old man with a history of Roux-en-Y gastricbypass and alcoholic cirrhosis complicated by ascites and esophageal varices status-post banding was transferred from an outside hospital for management of hepatic encephalopathy, which clinically improved with lactulose and rifaximin administration. He was recently diagnosed with acute on chronic kidney injury from presumed type 2 hepatorenal syndrome, and started on octreotide, midodrine, and albumin. His hospital course was complicated by persistent symptomatic hypoglycemia that required him to be on a continuous dextrose 50 percent in water infusion, and a high-carbohydrate diet.

Laboratory Studies: When the patient was symptomatically hypoglycemic with a blood glucose level of $43 \mathrm{mg} /$ $\mathrm{dL}$, he concurrently had elevated levels of serum insulin (25 mclU/mL), C-peptide (10.38 ng/mL), and pro-insulin (52 pmol/L).

Radiological Findings: Abdominal ultrasound and magnetic resonance imaging studies showed no gross evidence of a pancreatic mass. The initial somatostatin receptor scintigraphy showed increased activity in the region of the pancreatic head and uncinate process with focal uptake in the dome of the liver. However, a repeat somatostatin receptor scintigraphy about two weeks later showed resolution of the previously noted increased activity. Intra-arterial calcium stimulation with hepatic venous sampling showed no evidence of elevated insulin levels, significant vascular disease, or a hypervascular region. All of these studies were unrevealing for evidence of a neuroendocrine tumor.

\section{CLINICAL MANAGEMENT}

The patient was briefly treated with high-dose octreotide (up to $200 \mathrm{mcg}$ every 8 hour), hydrochlorothiazide, and diazoxide when the etiology of his hypoglycemia was thought to be a result of excess insulin secretion. The hydrochlorothiazide and diazoxide were discontinued in the setting of hyperkalemia and worsening kidney function. There was no appreciable improvement in the patient's blood glucose levels until the octreotide was discontinued and high-dose steroids was initiated. The patient was weaned off of all continuous dextrose infusion about three weeks after he last received octreotide, and was subsequently discharged home one week later on prednisone $5 \mathrm{mg}$ once daily. As an outpatient, the patient was tapered off of prednisone.

\section{DISCUSSION}

In the evaluation of hypoglycemia in patients without diabetes, it is essential to first distinguish between those that meet criteria for Whipple's triad from those who do not, as the former group requires further laboratory workup. Whipple's triad consists of a low plasma blood glucose concentration with concurrent symptoms of hypoglycemia, and symptomatic improvement after the blood glucose level is raised.

In our patient who had documentation of Whipple's triad, the differential for the etiology of his hypoglycemia included an insulinoma, nesidioblastosis, and side effect of octreotide administration. Nesidioblastosis after gastricbypass surgery causes hyperinsulinemic hypoglycemia as a result of pancreatic islet cell hypertrophy. ${ }^{3}$ Laboratory evaluation was consistent with those expected in an insulinoma given elevated levels of serum insulin, C-peptide, and pro-insulin when the patient's blood glucose level was $43 \mathrm{mg} / \mathrm{dL}$. However, repeat octreotide scans and portal venous sampling did not yield evidence of a neuroendocrine tumor.

Octreotide inhibits insulin secretion, and is thus indicated in the treatment of sulfonylurea-induced hypoglycemia. However, paradoxical hypoglycemia with octreotide use has been described in case reports of patients with an insulinoma and a proinsulinoma. ${ }^{4-7}$ In our patient, octreotide-induced hypoglycemia is the most plausible explanation for his persistent hypoglycemic state, which spontaneously resolved without additional intervention after discontinuation of octreotide. 


\section{CONCLUSION}

This is the first reported case of hypoglycemia from octreotide use in a cirrhotic patient being treated for hepatorenal syndrome. This rare side effect cannot be reliably predicted, thus necessitating close supervision and glucose monitoring during octreotide therapy.

\section{REFERENCES}

1. Enescu A, Petrescu F, Mitrut P, Petrescu IO, Padureanu V, Enescu AS. Hepatorenal Syndrome: Diagnosis and Treatment. Rom J Intern Med 2016;54(3):143-150.

2. Davenport A, Ahmad J, Al-Khafaji A, Genyk YS, Nadim MK. Medical Management of Hepatorenal Syndrome. Nephrol Dial Transplant 2012;27:34-41.

3. Service GJ, Thompson GB, Service FJ, Andrews JC, Collazo-Clavell ML, Lloyd RV. Hyperinsulinemic Hypoglycemia with Nesidioblastosis after Gastric-Bypass Surgery. NEJM 2005:353:249-54.

4. Stehouwer CD, Lems WF, Discher HR, Hackeng WH, Naafs MA. Aggravation of Hypoglycemia in Insulinoma Patients by the Long-acting Somatostatin Analogue Octreotide (Sandostatin). Acta Endocrinol (Copenh) 1989;121(1):34-40.

5. Gama R, Marks V, Wright J, Teale JD. Octreotide Exacerbated Fasting Hypoglycaemia in a Patient with a Proinsulinoma; the Glucostatic Importance of Pancreatic Glucagon. Clin Endocrinol (Oxf) 1995;43(1):117-20.

6. Healy ML, Dawson SJ, Murray RML, Zalcberg J. Severe Hypoglycemia After Long-acting Octreotide in a Patient with an Unrecognized Malignant Insulinoma. Internal Medicine Journal 2007:37:406-9

7. Abell SK, Teng J, Dowling A, Hofman MS, Maclsaac RJ, Sachithanandan N. Prolonged Life-Threatening Hypoglycemia Following Dose Escalation of Octreotide LAR in a Patient with Malignant Polysecreting Pancreatic Neuroendocrine Tumor. Endocrinol Diabetes Metab Case Rep 2015: 140097

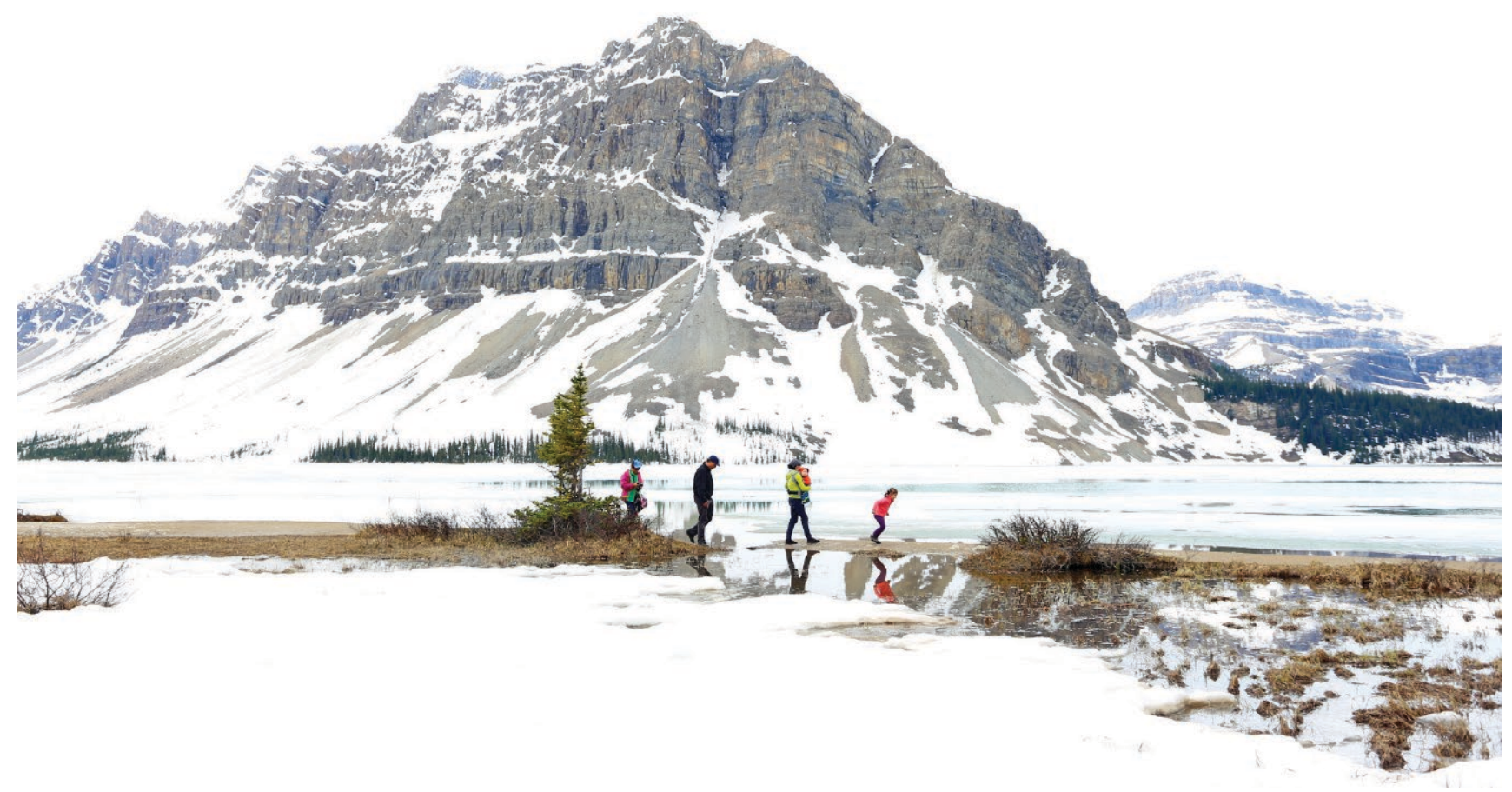

Sharon Li, MD 